\title{
Propuesta de un sistema de gestión integrado basado en NTC-ISO 9001:2015, NTC-ISO 14001:2015 y el Decreto 1072 de 2015 (capítulo 6) para una empresa de atención médica domiciliaria*
}

Proposal for an integrated management system based on NTCISO 9001:2015, NTC-ISO 14001:2015 and Decree 1072 of 2015 (chapter 6) for a home health care company

Proposta de um sistema de gestão integrado baseado na NTC-ISO 9001: 2015 NTC-ISO 14001:2015 e o Decreto 1072 (capítulo 6) de 2015 para uma empresa de atenção médica domiciliaria

Recibido: 20 de diciembre de 2015 Revisado: 30 de junio de 2016 Aceptado: 1 de agosto de 2016

Nidia Yisel Alfonso Zorro**

Centro de desarrollo Agroindustrial de Chía

* Artículo de resultado de investigación. DOI: http://dx.doi.org/10.15332/s2145-1389.2017.0001.03

** Magíster (c) en Calidad y Gestión Integral. Especialista en Gestión Integrada QHSE. Ingeniera Industrial. Líder del Sistema Integrado de Gestión del Centro de Desarrollo Agroindustrial de Chía (SENA). Correo electrónico: nidiayisel@hotmail.com. 
Sonia Stella Díaz Sánche*****

Zona Franca de Bogotá S. A.

Florangela Gómez Hernánde*******

Auditora independiente

\section{RESUMEN}

La investigación aborda los antecedentes de los sistemas de gestión integral para avanzar en la formulación de una propuesta del sistema de gestión integrado basado en las normas técnicas NTC-ISO 9001:2015 y NTC-ISO 14001:2015 y el Decreto 1072 de 2015 (capítulo 6) para una empresa de atención domiciliaria; desde un paradigma pragmático, dentro de una investigación mixta, luego de realizar el análisis cualitativo y cuantitativo de la situación actual, frente al grado de implementación de los sistemas de gestión en la entidad, se realiza una propuesta que brinda herramientas a la organización para establecer un sistema de gestión integrado.

Palabras clave: sistema integrado, gestión de la calidad, gestión ambiental.

\section{ABSTRACT}

The research addresses the background of comprehensive management system to advance the formulation of a proposal for the integrated management systems based on NTC-ISO 9001:2015, la NTCISO 14001:2015 and Decree 1072 of 2015 (chapter 6) for home care companies; from a pragmatic location, within a mixed research, after performing the qualitative and quantitative analysis of the current situation, compared to the degree of implementation of the management systems in the entity, a proposal is made that provides tools to the organization to establish an integrated management system.

Keywords: Integrated system, quality management, environmental management.

\section{RESUMO}

A investigação aborda os antecedentes dos Sistemas de Gestão Integral, para avançar na formulação de uma proposta do Sistema de Gestão Integrado baseado na NTC - ISO 9001: 2015, na NTC-ISO 14001: 2015 e o Decreto 1072 (Capítulo 6) 2015 para empresas de assistência domiciliária; desde um paradigma pragmático, dentro de uma pesquisa mista, após fazer uma análise quantitativa e qualitativa da situação atual em relação ao grau de execução dos Sistemas de Gestão na Entidade, foi feita uma proposta que oferece as ferramentas para que a organização possa estabelecer um sistema de Gestão Integrada.

Palavras-chave: sistema integrado, gestão, qualidade.

\section{INTRODUCCIÓN}

La investigación tiene como objetivo proponer un sistema de gestión integrado basado en la NTC-ISO 9001:2015, la NTC-ISO 14001:2015 y el Decreto 1072 de 2015 (capítulo 6) para la empresa de atención médica domiciliaria. Para cumplir con este fin, se

\footnotetext{
*** Magíster (c) en Calidad y Gestión Integral. Especialista en Gestión Integrada QHSE. Ingeniera Industrial. Analista Auditoria y Calidad de Zona Franca de Bogotá S. A. Correo electrónico: ingediaz7@hotmail.com.

**** Magíster (c) en Calidad y Gestión Integral. Fisioterapeuta. Auditora independiente. Correo electrónico: angelagoher@hotmail.com.
} 
determinaron como objetivos específicos la realización de un diagnóstico inicial para conocer el estado actual del sistema de gestión de la organización, respecto a las normas antes mencionadas, el cual permitirá formular una propuesta de sistema de gestión integrada, así como validarla por parte de los directivos de la organización y ajustarla conforme a los resultados que se obtengan de la evaluación.

La necesidad de este sistema de gestión integrado obedece a la existencia de varias problemáticas, siendo una de ellas que el tipo de contratación conlleva a una inestabilidad laboral de sus trabajadores, de ahí que se presente una rotación de personal constantemente, perdiendo información y retrasando sus procesos. Conforme a ello, la pregunta de investigación que se propuso es: ¿Cuáles son los elementos para una propuesta de un sistema de gestión integrado basado en la NTC-ISO 9001:2015, la NTC-ISO 14001:2015 y el Decreto 1072 de 2015 (capítulo 6) para la empresa de atención médica domiciliaria?

Para dar respuesta a este interrogante, la presente investigación aborda inicialmente los antecedentes de los sistemas de gestión integral, tomando en cuenta la normatividad nacional, la cual expresa:

En el año 1993 se publicó la Ley 100 en Colombia, cuyo objetivo principal ha sido garantizar la atención de salud de los ciudadanos del territorio nacional. Con esta ley se buscó ante todo cubrir las necesidades frente al estado de salud de las personas, sin tener en cuenta la capacidad económica de la población. (Ley 100, 1993)

De otra parte, se tienen en cuenta los esfuerzos del Congreso de la República de Colombia, puesto que en donde se generaron estrategias que permitieran el cumplimiento de esta ley, de ahí nace la atención médica domiciliaria; con el fin de reducir el hacinamiento de las instituciones de salud prestando un mejor servicio (Sierra y Díaz, 2010), para avanzar posteriormente en la descripción y formulación del problema, así como en la definición de la justificación y los respectivos objetivos, de otra parte, la presente investigación aborda antecedentes de los sistemas de gestión integral, como ejemplo, en 1947 se crea la International Organization for Standardisation (ISO), organización conformada por 163 países del mundo, la cual contaba con tres principios fundamentales: el primero, se basaba en las opiniones de las personas interesadas en la norma y su consenso; el segundo principio tiene como objetivo satisfacer las necesidades de las organizaciones y de los clientes y; finalmente, se habla de la participación voluntaria en la adopción de las normas planteadas por ISO (Lara, 2017).

Clarificados estos elementos, el trabajo continúa con el desarrollo del marco teórico, tomando en cuenta para ello las nociones de sistema integrado, ciclo PHVA, atención médica domiciliaria, sistema de gestión del ambiente, la NTC ISO 9001 de 2015 y el Decreto 1072 de 2015 (capítulo 6). Seguido a ello, se abordó el marco conceptual, buscando definir cada uno de los elementos mencionados anteriormente, pero a los que se les sumó los de liderazgo, comunicación, toma de decisiones y participación activa, entre otros.

Se continuó con la delimitación del marco constitucional y legal, partiendo de los postulados establecidos por la Carta Política y los decretos y leyes promulgados por el legislador y el ejecutivo en relación con los sistemas de gestión y los relativos a la atención médica domiciliaria. Una vez determinado el marco legal, el siguiente apartado trata sobre el diseño metodológico, el que se caracteriza por ubicarse desde una investigación pragmática, por otra parte, se aborda un enfoque mixto, los cuales desembocaron en el siguiente capítulo sobre análisis de resultados bajo estos dos enfoques, en el que se trataron cada una de las normas ISO, así como el decreto anteriormente mencionado en los objetivos de la investigación. 
Este análisis de resultados permitió desarrollar en el siguiente apartado una propuesta para la integración de los sistemas de gestión, desde las etapas de planeación estratégica, identificación e interacción de los procesos; hasta la construcción de una propuesta de metodología para la identificación de riesgos en los procesos. La propuesta de integración permitió que se llevara a cabo una validación de la misma, su respectivo ajuste y las conclusiones y recomendaciones que surgen por asumir un sistema de gestión integrado.

En síntesis, se plantea en el desarrollo del presente trabajo una propuesta de un sistema de gestión integrado que involucre un sistema de gestión de calidad, un sistema de gestión ambiental y un sistema de seguridad y salud en el trabajo, con el fin de dar cumplimiento a las normas establecidas y la implementación de posibles soluciones con base en un desarrollo sostenible.

\section{METODOLOGÍA}

La presente investigación se desarrolla dentro de una ubicación pragmática:

Ya que distingue los objetos reales del mundo y los teóricos (idealizaciones) de la ciencia, que describen a los otros. Así mismo, desplaza el acento negativo del realismo de Popper en la falsación de teorías hacia las contrastaciones superadas; si una teoría falla al resolver determinados problemas no es razón suficiente para descartarla. (Vásquez, Acevedo, Manasero y Acevedo, 2001, p. 9)

El tipo de investigación que se utilizó para el desarrollo del presente trabajo es mixto, "ya que implica un conjunto de procesos de recolección, análisis y vinculación de datos cuantitativos y cualitativos para responder al planteamiento del problema” (Hernández, Fernández y Baptista, 2014, p. 532).
Por una parte es cuantitativa, ya que para conocer el estado de la situación actual en la implementación de los sistemas de gestión bajo las nomas NTC-ISO 9001:2015, NTC-ISO 14001:2015 y el Decreto 1072 de 2015 (capítulo 6) que tienen la empresa de atención medica domiciliaria, las investigadoras utilizaron una lista de verificación que se organizó por capítulos, siguiendo la estructura de alto nivel que adoptó la ISO para la creación de las normas mencionadas anteriormente.

Por otra parte, es cualitativa, porque se "enfoca en comprender los fenómenos, explorándolos desde la perspectiva de los participantes en un ambiente natural y en relación con su contexto" (Hernández, Fernández y Baptista, 2014, p. 358). En la medida en que se interactuó con los miembros de la organización para la aplicación de la lista de verificación y así examinar la forma en que los individuos percibieron y experimentaron los fenómenos que los rodean para profundizar en sus percepciones.

De tal forma que al analizar la información obtenida por el diagnóstico que se realizó a la organización, se evidencia que existen requisitos que no han sido implementados.

El método que se utilizó en la investigación es deductivo, ya que parte de una serie de premisas en las que se pretende evidenciar el alcance de la implementación de los diferentes requisitos que exige un sistema de gestión integrado; partiendo de la aplicación del diagnóstico inicial en donde se observa el porcentaje de implementación del sistema integrado de gestión de la organización.

Este es el instrumento universal científico que permite conocer la realidad mediante la percepción de los objetos directos y fenómenos. 
A su vez, se implementa dentro de la investigación el diseño de la triangulación secuencial, que establece el cumplimiento de un objetivo para continuar con el otro. Bajo esta perspectiva, se inició con la implementación del diagnóstico, que dio lugar a la obtención de los resultados y análisis cualitativos y cuantitativos, para proceder enseguida con la elaboración de la propuesta de un sistema de gestión integrado basado en la NTC-ISO 9001:2015, la NTC-ISO 14001:2015 y el Decreto 1072 de 2015 (capítulo 6).

Finalmente, se realizó la validación de la propuesta elaborada para la cual se asignaron expertos en sistemas integrados de la empresa, quienes a través del instrumento de validación evaluaron, calificaron y realizaron observaciones para propiciar los respectivos ajustes.

Se realizó una validación por medio de la presentación al subgerente de calidad y TIC, la subgerente de Talento Humano, la dirección médica y la subgerente administrativa y financiera, quienes por su perfil profesional son conocedores y expertos de las normas NTC-ISO 9001:2015, NTC-ISO 14001:2015 y el Decreto 1072 de 2015 (capítulo 6), una vez realizada la sustentación de la propuesta, a través del formato de validación, que consta de diferentes preguntas asociadas a las etapas de la propuesta para la entidad, se hizo entrega a cada uno de los evaluadores para que dieran la calificación y observaciones. La calificación está en tres momentos adecuada a la organización (5 puntos), medianamente adecuada (3) y no adecuada (1). Con los resultados obtenidos se realizaron los ajustes pertinentes.

Se diseñó considerando el estado de madurez que tiene la organización en la implementación de las normas NTG-ISO 9001, NTC-ISO 14001 y el Decreto 1072 de 2015 (capítulo 6). Para realizar el diagnóstico inicial que consta de los requisitos de cada una de las normas que se integrarán y las respectivas preguntas asociadas a las mismas.

Se realizó un instrumento diagnóstico, el cual se construyó con todos los ítems de los requisitos de la NTCISO 9001, NTC-ISO 14001 y el Decreto 1072 de 2015 (capítulo 6), donde a cada pregunta relacionada con el requisito de la norma se le otorgó una calificación de 1 a 3 dependiendo del grado de cumplimiento. Una vez elaborado el instrumento, se realizaron entrevistas con el personal, en las instalaciones de la empresa.

Al implementar el instrumento se revisó la documentación existente de acuerdo a lo relacionado con las preguntas de los requisitos. Para la validación de la propuesta se implementó un instrumento de evaluación construido con preguntas asociadas a las etapas de la propuesta, como resultados se obtiene una puntuación y unas observaciones.

\section{RESULTADOS Y DISCUSIÓN}

El estado de la situación actual en la implementación del sistema de gestión bajo las nomas NTC-ISO 9001:2015, NTC-ISO 14001:2015 y el Decreto 1072 de 2015 (capítulo 6), permitió utilizar una lista de verificación que se organizó por capítulos, siguiendo la estructura de alto nivel que adoptó la ISO para la creación de las normas mencionadas anteriormente.

Esta lista de verificación consta de 470 preguntas y fueron aplicadas durante cinco sesiones al personal directivo de la compañía, entre los que se encuentran la subgerencia de calidad y TIC, la subgerencia de Talento Humano, la dirección médica y la subgerencia administrativa y financiera.

Posteriormente se organizó la información recolectada en dos momentos, por una parte la información 
cuantitativa que aborda los resultados de la norma NTC-ISO 9001:2015, NTC-ISO 14001:2015 y el Decreto 1072 de 2015 (capítulo 6); y de otra parte la información cualitativa que aborda el sistema de gestión de calidad y sus procesos, el contexto de la organización, la comprensión de las necesidades y expectativas de las partes interesadas, toma de conciencia, comunicación, etc.

Luego de realizar el análisis cualitativo y cuantitativo de la situación actual, frente al grado de la implementación de los sistemas de gestión en la entidad, bajo las normas NTC-ISO 9001:2015, NTC-ISO 14001:2015 y el Decreto 1072 de 2015 (capítulo 6), se realiza la siguiente propuesta con el objetivo de brindar una gestión integrada orientada a mejorar la calidad de sus servicios, obtener una mayor ventaja competitiva dentro del sector de atención médica domiciliaria, optimizar los procesos, minimizar los impactos ambientales, ofrecer mejores condiciones laborales y atendiendo las expectativas de las partes interesadas (empleados, proveedores, clientes, usuarios, comunidad y entes de control). Ello con el fin de lograr una mejor estabilidad en el mercado y mayor reconocimiento a nivel nacional. A continuación se enuncian las etapas que se consideraron para la propuesta:

\section{Etapa 1. Planeación estratégica}

Para determinar las cuestiones externas que son pertinentes a la razón de ser de la entidad, y teniendo en cuenta el direccionamiento estratégico, se utilizó para el análisis la herramienta PESTAL (que hace referencia a los aspectos políticos, económicos, sociales, tecnológicos, ambientales y legales), que permite evaluar de forma constante los elementos que pueden afectar o beneficiar a la empresa, dando a conocer el entorno en el que se opera.

Tabla 1. PESTAL

\begin{tabular}{|c|c|c|c|c|}
\hline Políticos y legal & Económicos & Sociales & Tecnológicos & Ambientales \\
\hline $\begin{array}{l}\text { Cambios legislativos } \\
\text { en salud. }\end{array}$ & $\begin{array}{l}\text { La poca inversión económica } \\
\text { del gobierno hacia el sector de } \\
\text { la salud. }\end{array}$ & $\begin{array}{l}\text { Domicilio del } \\
\text { paciente no apto para } \\
\text { la continuidad del } \\
\text { tratamiento domiciliario. }\end{array}$ & $\begin{array}{l}\text { Pocos conocimientos } \\
\text { tecnológicos de las } \\
\text { personas para el acceso } \\
\text { de la historia clínica para } \\
\text { obtener los medicamentos } \\
\text { que se requieren. }\end{array}$ & $\begin{array}{l}\text { No regulación del } \\
\text { consumo de energía. }\end{array}$ \\
\hline Monopolización. & $\begin{array}{l}\text { El retraso de los pagos de } \\
\text { las EPS hacia la empresa de } \\
\text { atención médica. }\end{array}$ & $\begin{array}{l}\text { Reacción negativa de } \\
\text { las personas frente al } \\
\text { servicio prestado por la } \\
\text { institución. }\end{array}$ & $\begin{array}{l}\text { Pocos avances de la } \\
\text { tecnología en Colombia } \\
\text { para el sector salud. }\end{array}$ & $\begin{array}{l}\text { No control del } \\
\text { reciclaje de desechos } \\
\text { orgánicos. }\end{array}$ \\
\hline $\begin{array}{l}\text { Licencias para la } \\
\text { ejecución de labores. }\end{array}$ & $\begin{array}{l}\text { Bajos recursos económicos de } \\
\text { las personas para afiliarse a } \\
\text { una EPS. }\end{array}$ & $\begin{array}{l}\text { Cambios en el estado de } \\
\text { salud de la persona. }\end{array}$ & Pérdida de la información. & \\
\hline $\begin{array}{l}\text { Cambios legislativos } \\
\text { para los prestadores de } \\
\text { servicios. }\end{array}$ & $\begin{array}{l}\text { La subida del dólar o } \\
\text { monopolización del mercado, } \\
\text { para la compra de equipos } \\
\text { médicos y medicamentos de } \\
\text { última tecnología. }\end{array}$ & $\begin{array}{l}\text { Mayores y mejores } \\
\text { ofertas en el mercado. }\end{array}$ & & \\
\hline
\end{tabular}

Fuente: elaboración propia 
Adicionalmente se utilizó la herramienta DOFA, la cual permite conocer la situación real de la organización, teniendo en cuenta los factores positivos y negativos, tanto internos como externos. Además, en la toma de decisiones oportunas y adecuadas a las situaciones que se puedan presentar, la matriz se divide de la siguiente manera: (D) Debilidades: se refiere a los aspectos negativos que se presentan dentro de la empresa; (0) Oportunidades: se define como las características externas que se pueden usar a favor en la empresa o para sus beneficios; (F) Fortalezas: son las características positivas de la empresa que permiten un mejor desempeño en sus labores; (A) Amenazas: son los acontecimientos externos, que pueden afectar el desempeño de la organización, a diferencia de las debilidades, es imposible generar controles por parte de la empresa (Francés, 2006).

\section{Matriz de debilidades, oportunidades, fortalezas y amenazas}

Tabla 2. DOFA

\begin{tabular}{|c|c|c|c|}
\hline Debilidades & Oportunidades & Fortalezas & Amenazas \\
\hline $\begin{array}{l}\text { 1. Personal no suficiente para } \\
\text { el desarrollo de las actividades } \\
\text { operativas. }\end{array}$ & $\begin{array}{l}\text { 1. Que las entidades prestadoras de } \\
\text { salud no cuenten con la infraestructura } \\
\text { necesaria para dar cubrimiento, y sea } \\
\text { necesario dirigir a los usuarios al servicio } \\
\text { médico domiciliario. }\end{array}$ & $\begin{array}{l}\text { 1. Se cuenta con un control } \\
\text { de inventarios donde se } \\
\text { maneja por sistema PEPS. }\end{array}$ & $\begin{array}{l}\text { 1. Que la organización no } \\
\text { adecúe sus equipos a los } \\
\text { cambios tecnológicos que se } \\
\text { presenten para la prestación } \\
\text { del servicio médico. }\end{array}$ \\
\hline 2. Alta rotación de personal. & $\begin{array}{l}\text { 2. Los usuarios no cuenten con fácil } \\
\text { acceso a las IPS y EPS ambulatorias. }\end{array}$ & $\begin{array}{l}\text { 2. Se cuenta con buena } \\
\text { señalización, acorde con la } \\
\text { normatividad vigente. }\end{array}$ & $\begin{array}{l}\text { 2. Poca accesibilidad frente } \\
\text { a los recursos tecnológicos } \\
\text { (software, hardware). }\end{array}$ \\
\hline $\begin{array}{l}\text { 3. Deficiencia en la asignación } \\
\text { de roles y responsabilidades. }\end{array}$ & $\begin{array}{l}\text { 3. La demanda es alta para la prestación } \\
\text { de atención médica domiciliaria. }\end{array}$ & $\begin{array}{l}\text { 3. Se cuenta con } \\
\text { profesionales certificados } \\
\text { y competentes al cargo a } \\
\text { desempeñar. }\end{array}$ & $\begin{array}{l}\text { 3. La vulnerabilidad que } \\
\text { presentan los trabajadores } \\
\text { en la prestación del servicio } \\
\text { durante el desplazamiento } \\
\text { para llegar al domicilio. }\end{array}$ \\
\hline $\begin{array}{l}\text { 4. Clima organizacional no } \\
\text { acorde que imposibilita la buena } \\
\text { prestación del servicio. }\end{array}$ & $\begin{array}{l}\text { 4. La preferencia de los usuarios a recibir } \\
\text { su tratamiento o prestación de servicio } \\
\text { de salud en casa, por comodidad. }\end{array}$ & $\begin{array}{l}\text { 4. Se está implementando } \\
\text { la formación de programas } \\
\text { que permite llegar a personas } \\
\text { paliativas, es decir, con más } \\
\text { vulnerabilidad. }\end{array}$ & $\begin{array}{l}\text { 4. Creación de nuevas IPS } \\
\text { con mejores servicios. }\end{array}$ \\
\hline $\begin{array}{l}\text { 5. Las autorizaciones no } \\
\text { son acordes con los tiempos } \\
\text { establecidos, dificultando la } \\
\text { prestación del servicio. }\end{array}$ & $\begin{array}{l}\text { 5. Preferencia de los pacientes y familias } \\
\text { de la atención médica domiciliaria por } \\
\text { atención personalizada. }\end{array}$ & $\begin{array}{l}\text { 5. Se realizan capacitaciones } \\
\text { al personal, permitiendo } \\
\text { mayor calidad en el servicio. }\end{array}$ & \\
\hline $\begin{array}{l}\text { 6. Equipos que no permiten } \\
\text { las especificaciones para la } \\
\text { prestación del servicio. }\end{array}$ & & $\begin{array}{l}\text { 6. Larga trayectoria en el } \\
\text { mercado de atención médica } \\
\text { domiciliaria. }\end{array}$ & \\
\hline $\begin{array}{l}\text { 7. No se cuenta con buena } \\
\text { seguridad en la información } \\
\text { (software, hardware). }\end{array}$ & & & \\
\hline
\end{tabular}

Fuente: elaboración propia 


\section{Etapa 2. Identificación e interacción de los procesos (caracterizaciones de los procesos, normatividad y requisitos aplicables)}

Se ajustó la representación esquemática de los procesos y la interacción de la organización, rediseñando los procesos misionales y ajustando los procesos de apoyo, teniendo como punto de partida la misión, visión, necesidades y expectativas de las partes interesadas. Se pretende dar una imagen humanizada del sistema de gestión concebido por la organización.

Asimismo, se estructuraron los procesos en tres líneas, teniendo en cuenta su funcionalidad e importancia: procesos estratégicos, procesos misionales y procesos de apoyo.

Los organigramas son representaciones gráficas que permiten conocer niveles de jerarquización, principales funciones y relaciones que existen entre cada cargo establecido. La principal función de los organigramas es analizar los cargos asignados, con el fin de conocer las fallas que se pueden presentar en la organización.

De acuerdo con las responsabilidades establecidas en la caracterización de procesos, se propone un nuevo enfoque teniendo en cuenta los niveles de jerarquización y cadenas de mando de los diferentes cargos de la organización, con el fin de presentar unidades de mando acordes a las necesidades de cada proceso, además de ofrecer un mayor control en algunos cargos y mejor equilibrio en la estructura organizacional.

\section{Etapa 3. Estructura organizacional (organigrama)}

De acuerdo a las actividades de la organización, se construyó una matriz que facilita la identificación de los posibles impactos ambientales para priorizar todos aquellos que generen mayor índice de afectación para la entidad, con el fin de tomar medidas preventivas de mitigación y las respectivas correcciones. La metodología planteada tuvo como base los conceptos establecidos en la norma NTC-ISO 14001:2015 (Conesa, 2010).

\section{Etapa 4. Identificación de aspectos e impactos ambientales}

Siguiendo las actividades de la organización, se construyó una matriz que facilita la identificación de los posibles impactos ambientales para priorizar todos aquellos que generen mayor índice de afectación para la entidad; con el fin de tomar medidas preventivas de mitigación y las respectivas correcciones. La metodología planteada tuvo como base los siguientes conceptos establecidos en la norma NTC-ISO 14001:2015 (Conesa, 2010): aspecto ambiental, impacto ambiental, valoración del impacto, significancia del impacto, procedimiento general para la identificación y evaluación de los impactos ambientales.

En la propuesta planteada, se tomaron en cuenta las escalas de calificación de la metodología adaptada por Conesa. 
Tabla 3. Metodología Conesa

\begin{tabular}{|c|c|c|c|}
\hline Criterio & Definición del criterio & Calificación & Valor \\
\hline \multirow{2}{*}{ CARÁCTER - CA } & \multirow{2}{*}{$\begin{array}{l}\text { Hace alusión al signo, es decir, si es beneficioso (+) } \\
\text { o perjudicial (-) de las distintas acciones que van a } \\
\text { actuar sobre los distintos factores considerados. }\end{array}$} & Positivo & $(+)$ \\
\hline & & Negativo & $(-)$ \\
\hline \multirow{5}{*}{ MAGNITUD - MA $\left(^{*}\right)$} & \multirow{5}{*}{$\begin{array}{l}\text { Se refiere al grado de incidencia de la actividad o } \\
\text { acción sobre el factor, en el ámbito específico en el } \\
\text { que actúa. }\end{array}$} & Baja & 1 \\
\hline & & Media & 2 \\
\hline & & Alta & 4 \\
\hline & & Muy alta & 8 \\
\hline & & Total & 12 \\
\hline \multirow{4}{*}{ COBERTURA - CO } & \multirow{4}{*}{$\begin{array}{l}\text { Se refiere al área de influencia teórica del impacto } \\
\text { en relación con el entorno total. }\end{array}$} & Puntual & 1 \\
\hline & & Parcial & 2 \\
\hline & & Extenso & 4 \\
\hline & & Total & 8 \\
\hline \multirow{6}{*}{ MOMENTO - MO } & \multirow{6}{*}{$\begin{array}{l}\text { El plazo de manifestación del impacto alude al } \\
\text { tiempo que transcurre entre el surgimiento de la } \\
\text { acción potencialmente impactante y el comienzo } \\
\text { del efecto de la misma sobre el factor del medio } \\
\text { considerado. }\end{array}$} & Crítico & $(+4)$ \\
\hline & & Inmediato (0) & 4 \\
\hline & & Corto plazo & 3 \\
\hline & & Medio plazo (1 a 10 años) & 2 \\
\hline & & Largo plazo (> 10 años) & 1 \\
\hline & & Crítico & $(+4)$ \\
\hline \multirow{3}{*}{ DURACIÓN - DU } & \multirow{3}{*}{$\begin{array}{l}\text { Es el tiempo que supuestamente permanecería el } \\
\text { impacto desde su aparición y a partir del cual el } \\
\text { factor afectado retornaría a las condiciones iniciales } \\
\text { previas a la acción. }\end{array}$} & $\begin{array}{l}\text { Fugaz: el impacto persiste por menos de un } \\
\text { (1) año }\end{array}$ & 1 \\
\hline & & $\begin{array}{l}\text { Temporal: si el impacto persiste entre } 1 \text { y } \\
10 \text { años }\end{array}$ & 2 \\
\hline & & $\begin{array}{l}\text { Permanente: la permanencia del impacto es } \\
>10 \text { años }\end{array}$ & 4 \\
\hline \multirow{4}{*}{$\begin{array}{l}\text { REVERSIBILIDAD } \\
-\mathrm{RV}\end{array}$} & \multirow{4}{*}{$\begin{array}{l}\text { Se refiere a la capacidad del medio socio-ambiental } \\
\text { para asimilar naturalmente un cambio o impacto } \\
\text { generado por una o varias actividades del proceso. }\end{array}$} & Corto plazo (< 1 año) & 1 \\
\hline & & Medio plazo (1 a 10 años) & 4 \\
\hline & & Largo plazo (entre 10 años y 15 años) & 8 \\
\hline & & $\begin{array}{l}\text { Irreversible: la recuperación natural de la } \\
\text { variable a su estado inicial, sin medidas de } \\
\text { manejo, no es posible o su tiempo es mayor } \\
\text { a } 15 \text { años }\end{array}$ & 12 \\
\hline \multirow{3}{*}{$\begin{array}{l}\text { REGUPERABILIDAD } \\
- \text { RC }\end{array}$} & \multirow{3}{*}{$\begin{array}{l}\text { Se refiere a la posibilidad de reconstrucción total } \\
\text { o parcial del factor afectado a consecuencia de la } \\
\text { actividad. }\end{array}$} & Recuperable de manera inmediata & 1 \\
\hline & & Corto plazo (< 1 año) & 2 \\
\hline & & Medio plazo (1 a 10 años) & 3 \\
\hline
\end{tabular}


Nidia Yisel Alfonso Zorro, Sonia Stella Díaz Sánchez, Florangela Gómez Hernández

\begin{tabular}{|c|c|c|c|}
\hline Criterio & Definición del criterio & Calificación & Valor \\
\hline \multirow{3}{*}{$\begin{array}{l}\text { REGUPERABILIDAD } \\
- \text { RC }\end{array}$} & \multirow{3}{*}{$\begin{array}{l}\text { Se refiere a la posibilidad de reconstrucción total } \\
\text { o parcial del factor afectado a consecuencia de la } \\
\text { actividad. }\end{array}$} & Largo plazo (entre 10 y 15 años) & 4 \\
\hline & & Mitigable, sustituible y compensable & 4 \\
\hline & & $\begin{array}{l}\text { Irrecuperable: el impacto no se puede } \\
\text { recuperar ni mitigar con MMA }\end{array}$ & 8 \\
\hline \multirow[t]{2}{*}{ Tipo (TI) - } & \multirow{2}{*}{$\begin{array}{l}\text { Se refiere a la relación causa-efecto o la } \\
\text { manifestación del efecto sobre una variable socio- } \\
\text { ambiental como consecuencia de una actividad. }\end{array}$} & $\begin{array}{l}\text { Directo: cuando el impacto que se está } \\
\text { evaluando es el resultado o consecuencia } \\
\text { principal de la actividad o acción que lo } \\
\text { genera }\end{array}$ & 4 \\
\hline & & $\begin{array}{l}\text { Indirecto: su manifestación no es } \\
\text { consecuencia directa de la acción }\end{array}$ & 1 \\
\hline \multirow{3}{*}{ PERIODICIDAD - PR } & \multirow{3}{*}{$\begin{array}{l}\text { Se refiere a la regularidad de manifestación del } \\
\text { efecto, bien sea de manera cíclica o recurrente. }\end{array}$} & $\begin{array}{l}\text { Continuo: permanece constante en el } \\
\text { tiempo }\end{array}$ & 4 \\
\hline & & $\begin{array}{l}\text { Periódico. Es intermitente a lo largo del } \\
\text { tiempo }\end{array}$ & 2 \\
\hline & & $\begin{array}{l}\text { Irregular y discontinuo: son variables, } \\
\text { irregulares o imprevisibles en el tiempo }\end{array}$ & 1 \\
\hline \multirow{3}{*}{$\begin{array}{l}\text { POSIBILIDAD DE } \\
\text { OCURRENCIA - PO }\end{array}$} & \multirow{3}{*}{$\begin{array}{l}\text { Se refiere a la probabilidad de que un impacto se } \\
\text { presente o no. }\end{array}$} & Baja & 1 \\
\hline & & Media & 2 \\
\hline & & Alta & 4 \\
\hline \multirow[b]{2}{*}{ ACUMULACIÓN - AC } & \multirow{2}{*}{$\begin{array}{l}\text { Da idea del incremento progresivo o no de la } \\
\text { manifestación de la alteración sobre la(s) variable(s) } \\
\text { socio-ambientales evaluadas }\end{array}$} & $\begin{array}{l}\text { Simple: cuando una acción no produce } \\
\text { efectos acumulativos }\end{array}$ & 1 \\
\hline & & $\begin{array}{l}\text { Acumulativo: al prolongarse la acción } \\
\text { generadora incrementa la gravedad del } \\
\text { impacto }\end{array}$ & 4 \\
\hline \multirow{3}{*}{ SINERGIA } & \multirow{3}{*}{$\begin{array}{l}\text { La sinergia se refiere a la acción de dos o más } \\
\text { causas cuyo efecto es superior a la suma de los } \\
\text { efectos individuales. }\end{array}$} & Sin sinergismo (simple) & 1 \\
\hline & & Sinérgico & 2 \\
\hline & & Muy sinérgico & 4 \\
\hline \multirow{9}{*}{$\begin{array}{c}\text { IMPORTANCIA } \\
\mathrm{I}=+\mathrm{CA}(3 \mathrm{MA}+2 \mathrm{CO}+\mathrm{MO} \\
+\mathrm{DU}+\mathrm{RV}+\mathrm{RC}+\mathrm{TI}+\mathrm{PR}+\mathrm{P} \\
\mathrm{O}+\mathrm{AC}+\mathrm{SI})\end{array}$} & \multirow{9}{*}{$\begin{array}{l}\text { El significado de la importancia de un impacto se } \\
\text { basa en la siguiente consideración: la importancia } \\
\text { (I) corresponde a la evaluación cualitativa del } \\
\text { impacto ambiental o el cambio generado sobre un } \\
\text { factor y su relevancia frente al estado del mismo. }\end{array}$} & \multicolumn{2}{|l|}{ Carácter negativo } \\
\hline & & Irrelevante o compatibles & Entre -14 a -24 \\
\hline & & Moderado & Entre -25 a -50 \\
\hline & & Severo & Entre -51 a -75 \\
\hline & & Crítico & Entre -76 a -100 \\
\hline & & Carácter positivo $(* * *$ & \\
\hline & & Reducido & $\mathrm{de}+11 \mathrm{a}+30$ \\
\hline & & Importante & $d e+31 a+55$ \\
\hline & & Muy importante & $>+55 a+88$ \\
\hline
\end{tabular}

Fuente: Conesa (2010) 
Propuesta de un sistema de gestión integrado basado en NTC-ISO 9001:2015, NTC-ISO 14001:2015 y el Decreto 1072 de 2015 (capítulo 6)...

\section{Etapa 5. Propuesta de metodología para la identificación de riesgos}

Aunque existen diferentes metodologías para gestionar los riesgos, una de las propuestas de este estudio consistió en diseñar para la organización la matriz de gestión del riesgo, la cual tuvo en cuenta las orientaciones de la norma ISO 31000, Modelo Estándar de Control Interno (MECI) y la Guía para la administración del riesgo del Departamento Administrativo de la Función Pública.

Para la construcción de esta herramienta, se consideraron variables que le permiten a la organización, de acuerdo a los procesos definidos y al objetivo de cada uno de ellos, incluir los eventos que se pueden presentar, la fuente que los genera, las causas que los originan, el contexto (interno, externo) establecido; facilitando así la identificación, análisis y evaluación del riesgo, el tratamiento a seguir y las fases de comunicación, monitoreo y revisión.

En la herramienta diseñada en formato Excel, se incluyó para la identificación del riesgo una lista desplegable que contiene la clasificación de los riesgos, con el fin de establecer con mayor facilidad el análisis del impacto. Entre los riesgos contemplados se encuentran: riesgo estratégico, riesgos de imagen, riesgos operativos, riesgos financieros, riesgos de tecnología y riesgos de cumplimiento.

Así mismo, se incluyó la fórmula matemática para realizar la calificación del riesgo. Es de resaltar que para el análisis del mismo, se tuvieron en cuenta cinco escalas para la probabilidad de ocurrencia y cinco para su impacto, considerando además el alcance en caso de materialización del riesgo.

La herramienta diseñada permite determinar la zona de riesgo (aceptable, moderado, inaceptable); se consideraron estas tres zonas teniendo en cuenta la eficacia de los controles existentes.

- Aceptable. Se asegura el resultado del proceso, el riesgo no se ha materializado y mediante la aplicación de estos controles se puede asegurar que el riesgo es controlable.

- Moderado. El control existente debe evaluarse, con el fin de garantizar el resultado satisfactorio del proceso mediante la mitigación del riesgo.

- Inaceptable. El control con el que actualmente se cuenta para la mitigación del riesgo no asegura que la materialización del mismo no se presente, por lo cual la entidad requiere adelantar las acciones inmediatas con el fin de asegurar la efectividad del control (establecer el control, reevaluarlo, establecer unos nuevos, entre otros).

La matriz permite realizar la valoración del control, determinando su eficacia y realizando una nueva valoración del riesgo.

Una vez la organización ha definido el tratamiento a seguir (responsables y fechas de ejecución) y ha definido la conveniencia de la acción realizada, la herramienta determina el nivel de riesgo residual.

La implementación de esta herramienta permitirá a la organización la identificación de los riesgos, enfocando sus esfuerzos para potencializar aquellos eventos positivos que se puedan generar; minimizando aquellos que traigan consecuencias negativas que afecten el logro de los objetivos.

Una vez desarrollada la metodología propuesta, la cual está basada en la mejora continua, se realizó un diagnóstico inicial para conocer el grado de madurez que tiene la organización frente al sistema de gestión de calidad ambiental y seguridad y salud en el trabajo; para el cual se estructuró un instrumento que incluye 
los requisitos contemplados en cada una de la normas mencionadas; para la aplicación de este instrumento, las investigadoras realizaron entrevistas con el personal directivo de la organización. Posteriormente, se tabuló y analizó la información con los siguientes resultados:

Etapa 1. Planeación estratégica: que contempla el contexto de la organización (utilizando la metodología PESTAL y DOFA), misión, visión, política, objetivos, matriz de indicadores, alcance del sistema integrado.

\section{Etapa 2. Identificación e interacción de los proce-}

sos: se realiza un representación gráfica de la interacción de los procesos, de igual manera, una descripción detallada de los mismos que incluye el objetivo y alcance del proceso, entradas, salidas, clientes, proveedores y una descripción de las actividades siguiendo el ciclo de mejora continua, requisitos aplicables, recursos necesarios para la realización del mismo, indicadores de gestión.

Etapa 3. Estructura organizacional: Teniendo en cuenta las responsabilidades y autoridades de los cargos se define el organigrama; con el fin de establecer líderes que den cumplimiento a los procesos y tengan autonomía para lograr el engranaje con los demás procesos.

\section{Etapa 4. Identificación de aspectos e impactos} ambientales: se construyó una matriz que facilita el registro de los aspectos e impactos ambientales para ser valorados a través de criterios de calificación.

Etapa 5. Propuesta de metodología para la identificación de riegos en los procesos: se diseñó una herramienta para el registro de los riesgos de procesos, la cual le permitirá a la organización documentar y evaluar de manera integral los riesgos a los que puede estar expuesta la organización y que pueden afectar el logro de sus objetivos.

\section{CONCLUSIONES}

Considerando los objetivos planteados en la investigación, se puede concluir que es importante comprender que este tipo de propuestas servirá para orientar las acciones que se vienen haciendo en la organización, lo cual permitirá evidenciar los beneficios del sistema, además al implementar el sistema de gestión integrado el cual está claramente orientado a los procesos y a la mejora continua, la organización logrará sus objetivos en la medida que tenga la habilidad para mantener la excelencia de sus procesos.

El sistema de gestión integrado proporciona un marco de trabajo para la mejora continua que permitirá a la organización mantener e incrementar la satisfacción de los usuarios, centrándose en sus necesidades y expectativas.

Con el ánimo de que la propuesta se ajustará a las características de la organización, se dejó a consideración a un grupo de expertos de la entidad quienes realizaron una validación, que bajo unos criterios establecidos, dieron sus apreciaciones. Se realizaron ajustes a la propuesta en los siguientes aspectos: misión, visión y matriz DOFA.

La implementación del sistema de seguridad y salud en el trabajo es una obligación dado el imperativo legal. Por esta razón, las entidades de atención domiciliaria toman la decisión de implementar el sistema mencionado. En ese sentido, es necesario reconocer que la ejecución adecuada de un sistema de gestión aporta a la competitividad, crecimiento económico y productivo de las organizaciones, siendo esto así, se podría implantar un sistema voluntario basado en la toma de conciencia y de pensamiento en la mejora continua, sin requerimientos de orden legal.

Implementar y mantener un sistema integrado de gestión hace posible armonizar los procesos, generar 
Propuesta de un sistema de gestión integrado basado en NTC-ISO 9001:2015, NTC-ISO 14001:2015 y el Decreto 1072 de 2015 (capítulo 6)...

bienestar en los trabajadores, dar un significado relevante al desempeño ambiental; de manera que permita responder de manera pertinente y eficiente a todos las partes interesadas.

No hay una consciencia natural para el cumplimiento de los requisitos de calidad, ambiental y de seguridad y salud en el trabajo, más allá de lo establecido por ley y de las exigencias de gobierno para el sector salud. La consecución de la información no es fácil, ya que no existe una cultura de coleccionar y recolectar datos.

\section{REFERENCIAS}

Conesa, V. (2010). Guía metodoloǵica para la evaluación del impacto ambiental. 4. ${ }^{a}$ ed. Madrid: Ediciones Mundi-Prensa.

Francés, A. (2006). Estrategias y planes para la empresa con el cuadro de mando integral. México: Pearson Prentice Hall.
Hernández, R., Fernández, C., y Baptista, P. (2014). Metodología de la investigación. 6.a. México: McGraw-Hill.

Lara, R. (2007). Sistema de gestión de la calidad basado en la norma internacional NTC-ISO 9001-2000. Bogotá: Escuela Judicial.

Ley 100. (23 de diciembre de 1993). Por la cual se crea el sistema de seguridad social integral y se dictan otras disposiciones. Diario Oficial n. 41.148. Recuperado de http://www.secretariasenado.gov.co/ senado/basedoc/ley__100_1993.html

Sierra, D., y Díaz, M. (2010). Plan de negocios atención domiciliaria en salud. Chía: Universidad de La Sabana, Facultad de Medicina.

Vásquez, A., Acevedo, J., Manasero, M., y Acevedo, P. (2001). Cuatro paradigmas básicos sobre la naturaleza de la ciencia. Recuperado de http://www.oei.es/ historico/salactsi/acevedo20.htm\#1 